\title{
GEO-C: ENABLING OPEN CITIES AND THE OPEN CITY TOOLKIT
}

\author{
C. Granell a , D. Bhattacharya ${ }^{b}$, S. Casteleyn ${ }^{a}$, A. Degbelo ${ }^{c}$, M. Gould ${ }^{\text {a,d }}$, C. Kray ${ }^{c}$, M. Painho ${ }^{\text {b }}$, S. Trilles ${ }^{\text {a }}$ \\ ${ }^{\text {a }}$ GEOTEC, Institute of New Imaging Technologies, Universitat Jaume I, Castellón, Spain - \\ (carlos.granell, sven.casteleyn, gould, strilles)@uji.es \\ ${ }^{\mathrm{b}}$ NOVA Information Management School, Universidade Nova de Lisboa, Lisbon, Portugal - \\ (dbhattacharya, painho)@novaims.unl.pt \\ c Institute for Geoinformatics, University of Muenster, Muenster, Germany - (degbelo, c.kray)@uni-muenster.de \\ d Environmental Systems Research Institute (ESRI), Redlands, CA, USA - mgould@esri.com
}

\section{Commission IV, WG IV/4}

KEY WORDS: Open cities, Smart cities, GIScience, participation, transparency, Open City Toolkit, doctoral programme, GEO-C

\begin{abstract}
:
The GEO-C doctoral programme, entitled "Geoinformatics: Enabling Open Cities", is funded by the EU Marie Skłodowska-Curie actions (International Training Networks (ITN), European Joint Doctorates) until December 2018, and is managed by three European universities in Germany, Portugal and Spain. 15 doctoral grantholders (Early Stage Researchers) were selected to work on specific three-year projects, all contributing to improving the notion of open cities, and specifically to an Open City Toolkit of methodologies, code, and best practice examples. Contributions include volunteered geographic information (VGI), public information displays, mobility apps to encourage green living, providing open data to immigrant populations, reducing the second-order digital divide, sensing of quality of life, proximity based privacy protection, and spatio-temporal online social media analysis. All doctoral students conducted long-term visits and were embedded in city governments and businesses, to gain experience from multiple perspectives in addition to the researcher and users' perspective. The projects are situated within three areas: transparency, participation, and collaboration. They took mostly a bottom-up (citizen-centric) approach to (smart) open cities, rather than relying on large IT companies to create smart open cities in a top-down manner. This paper discusses the various contributions to enabling open cities, explains in some detail the Open City Toolkit, and its possible uses and impact on stakeholders. A follow-up doctoral program has been solicited and, if successful, will continue this line of research and will strengthen aspects of privacy, data provenance, and trust, in an effort to improve relations between data (e.g. news) publishers and consumers.
\end{abstract}

\section{INTRODUCTION}

The EU-funded European Joint Doctorate "Geoinformatics: Enabling Open Cities" (GEO-C) targets a better understanding of the opportunities and achievements of GIScience to address smart and open city challenges from a variety of perspectives. GEO-C's overarching objective is to make substantial scientific progress towards the notion of smart (open) cities. The programme started in January 2015 and will run until December 2018. During the programme, 15 Early Stage Researchers (ESRs) were recruited and started their 36-month $\mathrm{PhD}$ projects related to the main theme of the programme: to contribute to the state of the art, at the intersection between GIScience (Goodchild, 2010; Roche, 2014) and open and smart cities. These ESRs joined a team of post-doctoral researchers distributed over three European universities that form the GEO-C consortium, namely University of Münster (Germany), Universidade Nova de Lisboa (Portugal), and Universitat Jaume I of Castellón (Spain). In total, a team of $30+$ doctoral and post-doctoral researchers within the GEO-C project are involved in exploring, researching and providing new avenues, methods and tools to realise smart and open cities, in which all groups of society can participate on all levels and benefit in many ways.

At the beginning of the GEO-C programme, a group of postdoctoral researchers and professors from the three universities published a position paper in which we pointed out six citizen-related challenges in current open and smart cities: the engagement of citizens, the improvement of citizens' data literacy, the pairing of quantitative and qualitative data, the need for open standards, the development of personal services, and the development of persuasive interfaces (Degbelo et al, 2016a). In that paper, we also identified methods and techniques from GIScience and argued that their use might tackle, or at least help to mitigate, the aforementioned challenges. Interested readers can refer to Degbelo et al. (2016a) for detail of the matching analysis between GIScience contributions in terms of methods, and tools to each of the six smart open cities challenges.

Two years later, the ESRs are in the last stage of their PhD projects. At this point, we take a step back and reflect on what we have accomplished within the GEO-C programme. In particular, we report on concrete ESRs research outcomes and results grouped into the six smart open cities challenges and discuss them in the context of what we stated two years ago. Did we fail to foresee the type of GIScience techniques and tools relevant for smart city challenges? Or were we successful with our forecast? In section 2 we attempt to answer this question.

Besides the training focus on the $15 \mathrm{PhD}$ research projects, the GEO-C program is a research project on its own. As such, its primary aim is to produce a joint-development of an Open City Toolkit (OCT), whose conceptualisation and design was initially presented and described in Degbelo et al. (2016a). In short, the

Corresponding author 
OCT was conceived as a way of transferring insights and solutions from GIScience to smart cities, which in practice became a way of transferring concrete ESR's components, applications, services, tools, and other types of research outputs to smart open cities. As we did with the ESRs' contributions, in this paper we describe the main tangible components and tools that shape the OCT and discuss them with respect to the initial ideas stated in Degbelo et al. (2016a). Are the OCT's components consistent with what was anticipated two years ago? What did we miss and why?

While Sections 2 and 3 summarise the overall progress made within the GEO-C programme over the last two years, and also briefly discuss the role of FOSS4G tools in the research outcomes and achievements, we examine in Section 4 the impact of the programme on different stakeholders. Indeed, the stakeholders are vital to evaluate the success of the GEO-programme and they have played a key role since the outset of the programme, both as associated partners of the programme and as potential end users of the GEO-C results. We discuss the impact of the OCT on stakeholders, commenting various use cases and scenarios in which different types of stakeholders and end users can benefit from the OCT. The primary goal of the GEO-C programme is to contribute to enabling smart open cities by proposing GIScience methods and tools collected into the "Open City Toolkit". In Section 5, we reflect on the extent to which that goal has been accomplished and point next steps to address promising open areas.

\section{RESEARCH CONTRIBUTION TO GISCIENCE AND} OPEN CITIES

Degbelo et al (2016a) discussed the importance of six citizenfocused challenges. Obviously, cities are complex entities and cannot be reduced to only six dimensions or challenges, and modern cities face many more challenges and pressing issues than the six identified in this work. Nevertheless, we specifically focused on challenges that were directly related to citizens' needs or affect them substantially. We intentionally did this based on the assumption that "smart cities cannot become a reality unless citizens are central actors in shaping their cities" (Degbelo et al. 2016a; Van den Bergh \& Viaene, 2015). In addition, as these challenges allowed us to explore and better understand the spatiotemporal interactions between cities and citizens, they are closely linked to GIScience tools and methods, which are a fundamental pillar in the GEO-C programme. The proposed six challenges classified in three main research areas are:

- Empowering Citizens:

- Deep participation, and

- Data literate citizenry

- Analytical Methods and Tools:

- Pairing quantitative and qualitative data, and

- Adoption of open standards

- Citizen-Centric Services:

- Personal services, and

- Persuasive interfaces

In what follows, we describe ESRs' individual contributions for each challenge, and briefly discuss them against original plans. Then, we comment the role of FOSS4G tools.

\footnotetext{
${ }^{1}$ ESRXX in brackets denoete the unique id of each ESR, as it can be found at http://www.geo-c.eu/people\#esr
}

\subsection{ESR's Individual Contributions}

2.1.1 Deep participation: It refers to "the design and execution of strategies to foster citizens participate at all levels" (Degbelo et al., 2016a), for example by means of raising awareness, building capacity and strengthening communities.

Three lines of research were planned (Table 4 in Degbelo et al. (2016a)). Central to deep participation is to identify and understand the main motivating factors that characterise online citizen participation. To this regard, Naranjo Zolotov (ESR05 ${ }^{1}$ ) investigates the determinants of the adoption of online citizen public participation (e-participation), which is the involvement of citizens in consultation and decision processes along with the government using ICT. Research models based on information systems and cognitive psychology theories have been proposed (Naranjo Zolotov et al., 2018a; Naranjo Zolotov et al., 2018b) to understand the factors that have an effect on the individual's behavioural intention to use e-participation. Those models were evaluated using quantitative methods (Naranjo Zolotov et al., 2018c) considering the structural equation modelling with data collected from citizens who experienced e-participation technologies.

A second line of research looked into the concept of Sense of Place, which is tightly linked to how citizens feel and perceive their surrounding environments. City managers normally use hierarchical administrative boundaries to deliver their policies and actions. For instance, participatory processes in planning decisions or decision-making processes about communal spaces are framed and regulated according to administrative boundaries. The underlying reason is the availability of census and socioeconomic data in those areas. Acedo's (ESR04) project is aimed at discovering alternatives to administrative boundaries for covering those participatory process. Contrary to static places in which might or might not cover people's significant places and relationships, alternative geographies based on dynamic areas that better represent people's feelings and perceptions were proposed (Acedo et al., 2017). Building upon some environmental psychology and social concepts such as sense of place and social capital, the proposed geographies define the spatial operationalization to incorporate geographies of sense of place into local urban planning interventions (Portela et al., 2018). Nevertheless, there is still a long way to go to relate what has been proven at the individual level to the community level to create real geographies of engagement in a smart city.

As public displays are becoming more pervasive in urban environments, they offer new opportunities for community engagement and participation (Hespanhol et al., 2015). Thus, a third line of research is being pursued by Du (ESR01). Her project focuses on using public displays for facilitating public participation and to elevate it from low levels to higher levels in an urban planning process. At the beginning of the research, a survey about public displays for public participation was conducted, discovering that current research on public displays is mainly targeting low levels of public participation (Du et al., 2017). The research question morphed naturally to fill this gap: how can interactive large public displays, i.e. Immersive Video Environments (IVE), be used to support higher level of public participation and consultation? An elicitation study followed to explore suitable interaction modalities for supporting public consultation via IVE. It led to two sets of user-defined gesture candidates and gave insights on how to realize public 
consultation on IVE. These preliminary results are promising to help interaction designers and developers create interactive public displays for enabling public interaction and consultation with respect to urban planning content.

The suggested contributions of GIScience to the challenge of deep participation (Table 1 in Degbelo et al. (2016a)) were generally confirmed (e.g. VGI, maps as a platform for spatial dialogue), although the level of sophistication explored has gone well beyond the proposed generalities then.

2.1.2 Data literate citizenry: It implies the definition of digital inclusion strategies and fostering data literate skills for all citizens to enable them to interpret and understand the processes and service that drive smart services.

One particular stream of research conducted by Bustamante (ESR03) aims to explore how mixed participatory approaches could be rethought in Human-Computer Interaction (HCI) to facilitate (young) forced migrants' engagement in the design of (geospatial) services supporting their (re)settlement. Initially, challenges and needs of (young) forced migrants upon arrival into an unfamiliar city were identified through a series of interviews. These included issues such as (young) forced migrants' limited knowledge of the local language, their collective ways for information access and sharing, and adult forced migrants occasional inexperience with digital (geospatial) services (Bustamante et al., 2018a). Subsequently, an active search for adaptations of participatory design approaches with young forced migrants was carried out, by combining it with core concepts and strategies (e.g., 'safe spaces', didactic reduction, reflective thinking) from participatory research, and didactics (Bustamante et al., 2018b). Results in terms of open-source prototype services were developed on the road towards transformations of digital (geospatial) services for and with young forced migrants.

The suggested idea of "Maps as one way of contextualizing and presenting primary data in an understandable way" (Table 1 in Degbelo et al. (2016a)) remained still valid but augmented with a series of participatory design approaches tailored to young forced migrants.

2.1.3 Pairing quantitative and qualitative data: It is about devising novel analytical techniques and tools to integrate data from diverse sources, both quantitative (objective measurements) and qualitative (subjective perceptions, opinions).

Four lines of research were planned (Table 4 in Degbelo et al. (2016a)). Air pollution is profoundly impacting the quality of life of the individuals in the city (Camarsa et al., 2014). It also plays a crucial role in developing policies and strategies to achieve the aims of environmental policies for ensuring healthy living in cities. GIS and spatial analysis methods have been traditionally used for air quality monitoring and analysis. However, one of the significant barriers to air quality monitoring at a city level is usually the scarcity of air quality monitoring network data. Gupta (ESR08) investigates approaches to enable air quality monitoring at the city level. More precisely, the research focuses on identifying the methods which can help in allowing the air quality monitoring using open data. The era of big data and crowdsourcing has opened up new possibilities to tackle such a problem (Gupta et al., 2018). Identifying optimal locations to place monitoring stations using Land Use Regression and using low-cost crowdsourcing for data collection is recognised as an interesting technique, which can guide in solving the air quality monitoring with less prediction errors at a city level.
A second line of research relates climate and atmospheric research communities, GIScience and smart open cities to investigate and develop climate downscaling procedures for urban areas (Smid \& Costa, 2017). Smid (ESR09) made substantial progress on climate data downscaling by developing and running climate simulations for major European cities to, eventually, produce a sort of ranking of European cities for future heatwave impacts (Smid et al., 2018). A remarkable outcome of the project is a set of curated datasets with simulated data generated by the execution of climate model ensembles. Examples are the dataset of decades-wise climate data scaled and curated for urban zones, and a second dataset that contains yearly simulated values derived from 8 different models for 8 indices over 30 European cities from 1971 to 2100.

A third type of project focuses on statistical analysis and predictions of the dynamics of urban mobility. Santa (ESR10) is working particularly on developing spatio-temporal statistical models for analysing, describing, and understanding urban dynamics framed as human activity and mobility. Input data sources are varied such as social media (number of geolocated tweets per area and per hour), city sensors (count of vehicles in the streets), public transport systems (flows origin-destination within a subway system), and call detail records from mobile phones. Implemented statistical models involve approaches from epidemic, functional, and graph data over repeated measurements for catching daily and weekly seasonality and describing common patterns as well as abnormal behaviours due to the occurrence of unexpected events.

Lastly, the fourth topic was centred on the analysis of spatiotemporal interactions of crime data to predict crime hotspots in cities. Moradi (ESR12) analyses events happening on a network such as traffic accidents, street crimes, and broken water pipes locations within a city. These events are georeferenced and may be represented as part of the network data model. For instance, traffic accidents are placed on the street's network. Thus, the aim is to discover how such events behave and to understand whether they are uniformly distributed over the space. Adaptive and non-adaptive methods have been defined like kernel smoothing and Voronoi estimates for hot-spot analysis (Moradi et al., 2017). As time is typical an intrinsic dimension of the events being analysed, the interaction and correlation of time and space are also of interest. Summary statistics such as Kfunction and pair correlation function allow to examine events interactions as for example if they tend to cluster or repulse over time, or remain randomly distributed.

Our forecast of GIScience contributions to the challenge of pairing quantitative and qualitative data (Table 2 in Degbelo et al. (2016a)) were not confirmed. For example, semantic-related techniques have been much less important than expected. In contrast, combined statistical and spatial analytical techniques have succeeded among the above ESRs.

2.1.4 Adoption of open data standards: Not only does it imply the use of standards in a broader sense to ensure interoperability, but it also refers to methodologies to promote the adoption of open data standards, conventions and policies to unlock the potential of all citizens to access and use open data.

Two lines of research were initially planned (Table 4 in Degbelo et al. (2016a)). The first one related to participatory sensing, which is gaining traction as new mobile technology developments allow people to take informed actions at the local level, providing capabilities for environmental monitoring, data aggregation and mining, as well as information presentation and 
sharing (Loreto et al., 2016). Khoi's (ESR06) research line was aimed to investigate, propose and develop a generic participatory sensing framework, especially addressing flexibility and usability requirements from non-expert users. Results from a comprehensive review on existing participatory sensing applications identified a set of requirements/features for the successful design and deployment of a participatory sensing framework. Some important requirements/features include user privacy, multi-purpose, real-time visualization of the results, ability to give feedback to the participants, and incentive mechanisms. Subsequently, a prototype was built incorporating the identified requirements/features (Khoi et al., 2017) and deployed in real-world scenarios. These deployments seek to verify the usability of the framework and examine how participants of diverse backgrounds react to the features implemented in the framework (e.g., incentive, contextawareness). Preliminary results show that the framework is well understood and appreciated by the participants who positively react to the different incentive mechanisms embedded in the framework.

Open (Geographic) Data has been widely recognized as the engine to promote transparency, collaboration and participation, especially when applied to (open) cities. The second line of research focuses precisely on the improvement of reusability level of open geographic data at local level (Benitez-Paez; ESR11). As two cities never have the same socio-economiccultural substrate, the identification of the actual barriers that limit geographic data reuse in a city is a prerequisite prior to any intervention. A research contribution has been the definition of a taxonomy of barriers, obstacles and issues for data reuse considering a large pool of users' perceptions and requirements that inform local authorities for the definition of pertinent actions to re-shape and boost open data strategies (Benitez-Paez et al., 2018). Next, the analysis of empirical data collected through participatory workshops and traditional methods like surveys and questionnaires was crucial to develop a conceptual framework to overcome such identified obstacles and propose successful routes to improve the reusability levels of open geographic data in cities (Benitez-Paez et al., 2018b).

Our forecast in giving so much importance to the suite of Open Geospatial Consortium (OGC) standards was exaggerated (Table 2 in Degbelo et al. (2016a)). While this assumption may yet be valid in theory, in practice ESRs took different approaches to deal with open city data, demonstrating that OGC standards are only one type among the myriad of standards one can encounter in open city data portals.

2.1.5 Personal services: It denotes the design, exploration and development of customised and focused services that are able to adapt to the peculiarities and needs of individual citizens.

Two lines of research were initially planned (Table 4 in Degbelo et al. (2016a)). The first one emphasised the need for ensuring privacy protection for developing personal, trusted services. Ataei (ESR13) explores this by designing Location-Based Services (LBS) with the focus on protecting location privacy of users. Location information is essential to LBS, but it also has the potential to reveal sensitive information to malicious agents. To preserve location privacy, ESR13 aims to find methods to integrate location privacy protection in the process of LBS development. A conceptual model to address location privacy issues in the design and development process of LBS from both legal and technical perspectives was developed (Ataei \& Kray, 2017). In particular, this model analyses the role of location data management in the context of privacy preservation, and proposed the concept of temporal and spatial ephemerality to improve location privacy in the context of a location-based service. In addition, an ongoing development will put in the users' hands a tool to get a better understanding of location privacy and make informed choices to control their location privacy.

While smart cities rely on ubiquitous and context-aware computing to offer an intelligent digital environment, the dynamic communication between mobile devices remains a challenge. A second line related to personal services explores synergies between mobile devices, context-aware systems (Alegre et al., 2016), and social behaviour and ontologies (Atzori et al., 2012). Kamberov (ESR14) explores the application of social roles and relationships to the world of context-aware, location-based mobile devices to enable opportunistic communication among nearby devices and/or services. The approach relies on an ontology model that exploits social concepts and role theory to permit such an opportunistic communication among devices (Kamberov et al., 2017). Usecase scenarios using the afore-mentioned model were developed (Kamberov et al., 2018) as a basis to proposed software engineering and algorithmic solutions for developing a proof-ofconcept tool and services.

The emphasis was put on location-based services and research on location privacy (Table 3 in Degbelo et al. (2016a)). Whereas a project has been devoted to address location privacy issues, the other project explored the addition of a social layer into mobile devices to enable location-based, opportunistic communication, which might be regarded as a new generation of LBS and apps.

2.1.6 Persuasive interfaces: It explores novel, technologymediated ways to engage with citizens and to involve them in decisions that affect them.

Two lines of research were initially planned (Table 4 in Degbelo et al. (2016a)). The first line involved the use of geospatial technology, location-based services and visual interfaces for enhancing green behaviour and/or living. In this context, it is clear that sustainable, green transport modes in cities is unavoidably a central issue to decision makers, urban planners and city managers. Consequently, Pajarito's (ESR07) research topic considers the struggle between advocacy groups lobbying for increasing the use of bicycles and, on the other hand, citizens who do not easily change their transport routines and behaviour to fully embrace cycling. There is a clear need to leverage persuasive technologies, such as gamification, for encouraging the use of bicycles for commuting (Pajarito \& Gould, 2017). For doing so, an open source mobile geo-game app for recording bicycle trips and rewarding cyclists based on collaboration and competition strategies was designed, developed and tested in real settings. It allowed to evaluate its impact on cyclists' satisfaction, engagement, and intentions to cycle in three different European cities (Münster, Germany; Castellón, Spain; and Valletta, Malta). The results showed that collaboration-based rewards produced higher impacts on satisfaction and cycling engagement while feedback from participants provided insights for developing persuasive strategies for urban cyclists. Resulting open datasets of bicycle trips can serve to perform advance spatial analysis of cycling conditions and to evaluate spatial analysis tools for describing cycling mobility based on GPS tracks.

The second line of research aimed to explore and analyse the social implications of geospatial technology and location-aware interfaces for promoting affection, empathy and behaviour changes. Portela's (ESR15) research topic can be summarised as improving citizens' awareness in the urban space. This topic is 
particularly focused on the geographies of empathy as a means of how the relationship with other humans and non-humans can be traced in varied manners (Portela \& Granell-Canut, 2016). Both representational models (e.g. maps, digital data) and nonrepresentational styles of observing affective life are used to account for the implications and political assemblages that rule the world of the mundane in the urban and non-urban life. Examples of experiments include the study of emphatic traces in the interaction with chatbots (Portela \& Granel-Canut, 2017a), the observation of street level interactions with public objects (Portela \& Granell-Canut, 2017b), and the experimentation with technological and lighting installations that play with people's senses in the urban infrastructure. These interventions pursue to better understand how physical and imaginative realities jointly work in the perception of daily life activities.

We identified the spatialisation of user interfaces as key in the realisation of persuasive interfaces (Table 3 in Degbelo et al. (2016a)). Despite the fact that this GIScience technique matters, both research projects made use of additional methods such as game elements, gamification strategies and diverse social science techniques to engage citizens and provoke behaviour change.

\subsection{Relation to FOSS4G}

The GEO-C program is part of the Open Data pilot promoted by the European Commission to make accessible any type of research outcome other than scientific publications (journals, conferences), especially software artefacts and data (e.g. survey results, data collected in experiments). Other types of resources have been produced such as conceptual methodologies and frameworks, but software and data represent by far the most research outputs within the GEO-C programme.

Source code of software outcomes, be applications, libraries, services, or mobile apps, are available for download in the project account in GitHub: https://github.com/geo-c. Related to FOSS4G, projects within the areas of Empowering Citizens and Citizen-centric Services use conventional, well-known FOSS4G tools (QGIS, Postgresql, PostGIS) combined with other commercial environments like ArcGIS. For those projects that have produced mobile apps and/or web applications, Java/Android was the preferred programming language/platform for the former while the later ones chose JavaScript and the suite of third-party (geospatial) libraries (e.g. JQuery, Leaflet, Node, Express).

It is not a surprise that projects involved in the Analytical Methods and Tools theme are more demanding in the usage of sophisticated FOSS4G and data analysis tools. R (RStudio, extRemes R packages, Shiny) became the tool of choice, accompanied by well-established spatial analysis libraries and tools like QGIS and GRASS. Specific needs were covered by specialised tools such as Climate Data Operator, NcView, Panoply, etc.

Some critical voices claimed that the GIScience community needs an innovation platform to deploy and validate new ideas, and empower geographical research more broadly, as R does in the research in statistics (Gahegan, 2018). Nevertheless, our results demonstrate that research in GIScience can be done with a multitude of libraries and tools (e.g. R, D3, GRASS, QGIS, PostGIS) that ESRs used for their experiments instead of a single codebase.

\section{THE OPEN CITY TOOLKIT}

The Open City Toolkit (OCT) was defined in Degbelo et al. (2016a) as "a collection of tools, processes, specifications and guidelines to empower citizens to participate in and shape the future of their cities, and to deliver services based on open data that are useful for citizens, businesses and governing bodies alike". This definition is general enough to remain valid today, but we can refine it in practice as a way to gather the resulting contributions, whether or not derived from GIScience (see Section 2.1), within the GEO-C programme for the benefit of open cities. In addition, we imagined the realization of the OCT with a dual role in mind: as a platform and as a repository/catalogue. While the latter has been largely achieved, we were only partially successful in realising the platform role of the OCT as initially planned. We discuss this later in Section 5. Yet, the OCT contains components, as explained below, independent from the ESRs' contributions, which still play a key role to support the integration of existing libraries, data and services into operational applications delivered to end users.

We also envisioned five components of the OCT and their roles (Degbelo et al., 2016a), informed by a brief analysis and assessment of similar toolkits for open cities (Degbelo et al., 2016c). For each component, we add the research challenge mentioned in Section 2 in brackets:

- A set of tools to improve transparency (deep participation and data literate citizenry);

- A curated set of examples of open source apps, open data and services (pairing quantitative and qualitative data, personal services, and persuasive interfaces);

- An abstract architecture (open standards);

- A "glue" API to connect resources, apps and services;

- A tool for creating interactive guidelines on how to realize an open city.

In the remainder of this section, we summarise the status of the components that finally constitute the OCT, which have evolved from the list above. We comment their relation to FOSS4G too.

\subsection{OCT Catalogue}

As presented in Degbelo et al. (2016d), one of main parts of OCT is a catalogue. It is the entry point to access all ESRs' individual research resources and the rest of self-developed components and tools that constitute the OCT (see below). In terms of functionality, the OCT catalogue has the ability to provide all the complementary but much-needed functionality to support the discovery, browsing, access, edition, and visualization of OCT resources. A CKAN-based implementation met our requirements since CKAN comes with built-in facilities for managing, browsing, and exploring resources. We have used existing CKAN extensions, customised them, and integrated them into the live instance of the OCT catalogue (http://giv-oct.unimuenster.de:5000). Examples of installed extensions are data viewers (pdf, image and text), chart viewers using different visualizations, RDF vocabularies, local storage, API for reading and connecting to google analytics, usage statistics, and the creation of documentation pages.

As our interest lies in extensions which are able to manage and handle geospatial data and resources, the OCT catalogue has a strong relation to FOSS4G. For instance, to show and retrieve well-known GIS data, it uses the spatialUI, spatial metadata, spatial query, geo view and geojson view for visualising it. 


\subsection{Semantic API and OCT Transparency tool}

The Transparency tool aims to provide re-use information about open datasets, i.e., which applications use what datasets and how frequently do they use them. The Transparency tool monitors the access and use of public datasets in order to inform data portal managers and citizens about the current usage and impact of the published data access. Therefore, it is a highly useful tool to measure the impact of a city data portal by looking at the most frequently used datasets. In addition, the tool can provide insights regarding where to focus curation efforts to ensure the efficiency of derived data-driven public services. Examples of web maps, built using open source technologies, and making use of the transparency tool were recently presented (Degbelo \& Kauppinen, 2018).

An essential technical means of realizing this is the use of semantic Application Programming Interfaces (APIs). The design of semantic APIs and their different layers were discussed in detail in Degbelo et al. (2016b). In a nutshell, the semantic API helps to retrieve datasets according to their type (e.g., sensor, climate, finance), and facilitates reasoning about and learning from them.

\subsection{Interactive Guidelines Tool}

The final component of the OTC is the Interactive Guidelines tool (http://elcano.init.uji.es/guidelines). This tool supports the creation of guidelines to help city stakeholders by providing walk-through guided by a narrative or use case scenario. These guidelines refer to problem-solution patterns by presenting challenges, benefits and impacts in an understandable manner, i.e., everyone may share and refer to them when talking to others about these guidelines. The qualifier "interactive" underlines the ability of users to dynamically explore and interact (to certain degree) with the guideline through a set of blocks for different purposes such as graphs and plots creation, maps visualisation, custom JavaScript code and p5 code (a sort of JavaScript wrapper for Processing), and the inclusion of text and markdown formats.

These interactive elements/blocks are able to handle data sources, both deployed in the OCT catalogue (or any CKAN-based instance platform) and published elsewhere. For example, a graph can take as input a (raw) data source available in the OCT catalogue, permitting users to interact with the graph, and thereby with the associated data source. Furthermore, any resource registered in the OCT catalogue is susceptible to being an input data source for interactive guidelines by only specifying its access point (e.g. URL).

The Interactive Guidelines tool makes use of FOSS4G to support map-based visualisations for map and $\mathrm{p} 5$ blocks. The former relies on the Leaflet library while the latter integrates Mappa.js, a JavaScript library that allow to overlay a canvas on top of a tile map (https://github.com/cvalenzuela/Mappa).

\section{IMPACT OF THE OCT ON STAKEHOLDERS}

The effectiveness of the OCT towards providing support to the stakeholders came to the forefront on the occasion of meetings and discussions we had with them. Next, we briefly summarise some example of ESRs' contributions accessible though the OCT catalogue.

The impact of the WebGIS platform for urban climate downscaling was appreciated by municipal and industrial partners. The rich datasets prepared for climate modelling attracted interest from data analytics companies. The development of place attachment and sense of place concepts fascinated local administrators in municipalities who were willing to utilizing the methods, information and knowledge in a workable way for cities; if a practical proposal could be set up such as the demonstration of an online application at placeandcity.com, then even the Lisbon Municipality could be interested in developing a work plan. For furthering the study of digital divide and adoption of technology, the prospects of applications of artificial intelligence, block chain, data analytics into the developed tools also attracted the interests of stakeholders. As regards the tools to analyse urban dynamics, mobility and trends in social data, potential application areas that interested all stakeholders ranged from health data analysis apps, to human activities projections, to social behaviours and their practical implementations.

The OCT was designed to adopt an inclusive approach, to engage varied groups of stakeholders, including for example citizens, city councils, private companies, software developers, and researchers, to make their cities more liveable (Degbelo et al., 2016d). While the OCT initially targeted these profiles, some of them have shown more interest such as city councils and local public agencies/departments (e.g. police, urban planners), and even a few more emerged during the course of the GEO-C programme (e.g., young migrants and refugees. see section 2.1.2).

\section{REFLEXIONS AND OUTLOOK}

This paper summarizes the work done within the GEO-C programme, which aimed to bring GIScience insights and methods to the equation for smart and open cities. In a self-reflective exercise, we attempted here to compare what we initially envisioned at the beginning of the project, and what we finally accomplished. Though not final (as the programme is still ongoing until the end of 2018), we provided some insights regarding successes, failures and issues we encountered.

In terms of ESR individual resources, we discussed deviations from the initial plans in Section 2. While the initial specifications of the ESRs' topics were described in broad terms, the subsequent research activities have been narrowing down to necessarily focus on specific, limited areas, as usually occur in $\mathrm{PhD}$ projects.

Regarding the OCT, the achieved results are generally consistent in terms of individual components and tool developed (Section 3). However, we observe that the role of the OCT as an integrative platform does not reach out initial expectations. Yet, substantial work has been done to establish the OCT well beyond of a mere catalogue of disconnected outputs. For example, synergies were encouraged between ESRs resulting in joint publications which often also involved the combination of different outputs and tools proposed by ESRs. In some cases, software outputs have been reused to successfully achieve internal collaboration among ESRs. Obviously, our initial ambition was that each resource deployed into the OCT was potentially reusable by external city actors and stakeholders on the road to envision transparent and open cities. While internal collaboration and re-use of separately developed techniques and tools is promising, it is not clear to which extent such integration will be externalizable, and thus, to what degree our initial goal will be reached by the end of the programme.

Smart and open cities are intrinsically spatial entities (Roche, 2014). But cities cannot fully evolve considering only the spatial 
dimension. Cities are multi-dimensional, living and complex entities that are continually changing (Batty et al., 2012). GIScience does not facilitate coping with all the complexity of cities but can be an integral part of mainstream smart city technology and solutions along with social and citizens-driven factors. A recent compilation of 20 cases (Word Economic Forum, 2017) shows how cities around the word have achieved deeper insights on open and smart cities as a result of combining recent advances in technology, data collection and analysis to improve public services and liveability for their citizens. This is also the ultimate goal of GEO-C programme, to encourage city dwellers to become first-hand participants in the improvement of their cities. A follow-up doctoral program has been solicited and, if successful, will continue this line of research and will strengthen aspects of privacy, data provenance, and trust, in an effort to improve relations between data (e.g. news) publishers and consumers.

\section{ACKNOWLEDGEMENTS}

The authors gratefully acknowledge funding from the European Commission through the GEO-C project (H2020-MSCA-ITN2014, Grant Agreement Number 642332, http://www.geo-c.eu/). Sven Casteleyn and Carlos Granell are funded by the Ramón y Cajal Programme (grant numbers RYC-2014-16606 and RYC2014-16913, respectively). Sergi Trilles has been funded by the postdoctoral programme Vali+d (GVA) (grant number APOSTD/2016/058). Lastly, we sincerely thank to all ESRs who have been crucial for the success of the GEO-C programme.

\section{REFERENCES}

Acedo, A., Painho, M., and Casteleyn, S., 2017. Place and city: Operationalizing sense of place and social capital in the urban context. Transactions in GIS, 21(3), pp. 503-520.

Alegre, U., Augusto, J. C., and Clark, T., 2016. Engineering context-aware systems and applications: A survey. Journal of Systems and Software, 117, pp. 55-83.

Ataei, M., and Kray, C., 2017. Ephemerality Is the New Black: A Novel Perspective on Location Data Management and Location Privacy in LBS. In Progress in Location-Based Services 2016. Lecture Notes in Geoinformation and Cartography, Springer, pp. 357-373.

Atzori, L., Iera, A., Morabito, G., and Nitti, M., 2012. The Social Internet of Things (SIoT) - When social networks meet the Internet of Things: Concept, architecture and network characterization. Computer Networks, 56(16), pp. 3594-3608.

Batty, M., Axhausen, K. W., Giannotti, F., Pozdnoukhov, A., Bazzani, A., Wachowicz, M., Ouzounis, G., and Portugali, Y., 2012. Smart cities of the future. European Physical Journal: Special Topics, 214, pp. 481-518. https://doi.org/10.1140/epjst/e2012-01703-3.

Benitez-Paez, F., Degbelo, A., Trilles, S., and Huerta, J., 2018a. Roadblocks hindering the reuse of open geodata in Colombia and Spain: A data user's perspective. ISPRS International Journal of Geo-Information, 7(1), 6.

Benitez-Paez, F., Comber, A., Trilles, S., and Huerta, J., 2018b. Creating a conceptual framework to improve the re-usability of open geographic data in cities. Transactions in GIS (in press). https://doi.org/10.1111/tgis.12449.

Bustamante Duarte, AM., Degbelo, A., Kray, C. (2018a). Exploring Forced Migrants (Re)settlement \& the Role of Digital
Services. The 16th European Conference on ComputerSupported Cooperative Work (ECSCW). Nancy, France. June 48, 2018.

Bustamante Duarte, AM., Brendel, N., Degbelo, A., Kray, C. (2018b). Participatory Design and Participatory Research: An HCI Case Study with Young Forced Migrants. ACM Transactions on Computer-Human Interactions, 25, 1, Article 3, 39 pages. https://doi.org/10.1145/3145472

Camarsa, G., et al., 2014. LIFE and air quality. DirectorateGeneral for Environment, European Commission. Publications Office of the European Union. http:// dx.doi.org/10.2779/13976.

Degbelo, A., Granell, C., Trilles, S., Bhattacharya, D., Casteleyn, S., Kray, C., 2016a. Opening up Smart Cities: Citizen-centric Challenges and Opportunities from GIScience. ISPRS International Journal of Geo-Information, 5:16.

Degbelo, A., Trilles, S., Kray, C., Bhattacharya, D., Schiestel, N., Wissing, J., and Granell, C., 2016b. Designing semantic APIs for open government data. JeDEM - eJournal of eDemocracy and Open Government, 8(2), pp. 21-58.

Degbelo, A.; Bhattacharya, D.; Granell, C.; and Trilles, S., 2016c. Toolkits for smarter cities: a brief assessment. In: Ubiquitous Computing and Ambient Intelligence. Canary Islands, Spain, Nov 2016. Springer, vol. 10070, pp. 431-436.

Degbelo, A., Granell, C., Trilles, S., and Bhattacharya, D., 2016d. D8.2 Open City Toolkit Outline. Technical Report D8.2, http:/geo-c.eu (30 April 2018).

Degbelo, A., and Kauppinen, T., 2018. Increasing transparency through web maps. In $W W W$ '18 Companion: The 2018 Web Conference Companion. Lyon, France: ACM.

Du, G., Degbelo, A., Kray, C., 2017. Public displays for public participation in urban settings: a survey. In 6th $A C M$ International Symposium on Pervasive Displays. Lugano, Switzerland, ACM, https://doi.org/10.1145/3078810.3078825.

Gahegan, M., 2018. Our GIS is too small. The Canadian Geographer, 62(1), pp. 15-26. https://doi.org/10.1111/cag.12434

GEO-C project consortium, 2018. Joint Doctorate in Geoinformatics: Enabling Open Cities. European Commission Marie Skłodowska-Curie Actions, European Joint Doctorates (EJD). http://geo-c.eu/ (30 April 2018).

Goodchild, M.F., 2010. Twenty years of progress: GIScience in 2010. Journal of Spatial Information Science, 1, pp. 3-20.

Gupta, S., Mateu, J., Degbelo, A., and Pebesma, E., 2018. Quality of life, big data and the power of statistics. Statistics \& Probability Letters. https://doi.org/10.1016/j.spl.2018.02.030

Hespanhol, L., Tomitsch, M., McArthur, I., Fredericks, J., Schroeter, R., and Foth, M., 2015. Situated interfaces for engaging citizens on the go. Interactions, 23(1), pp. 40-45. https://doi.org/10.1145/2851200

Kamberov, R., Granell, C., Santos, V., 2017. Sociology Paradigms for Dynamic Integration of Devices into a ContextAware System. Journal of Information Systems Engineering \& Management, 2(1), 2.

Kamberov, R., Granell, C., and Santos, V., 2018. Use Case Scenarios of Dynamically Integrated Devices for Improving 
Human Experience in Collective Computing. In: Trends and Advances in Information Systems and Technologies (WorldCist 2018). Naples, Italy. Springer, vol. 746, pp. 581-592.

Khoi, N.M., Rodríguez-Pupo, L.E., and Casteleyn, S., 2017. Citizense-A generic user-oriented participatory sensing framework. In: Selected Topics in Mobile and Wireless Networking (MoWNeT 2017), pp. 1-8.

Loreto, V., Haklay, M., Hotho, A., Servedio, V. D., Stumme, G., Theunis, J., and Tria, F. (Eds.), 2016. Participatory Sensing, Opinions and Collective Awareness. Springer.

Moradi, M. M., Rodríguez-Cortés, F. J., and Mateu, J., 2017. On kernel-based intensity estimation of spatial point patterns on linear networks. Journal of Computational and Graphical Statistics. https://doi.org/10.1080/10618600.2017.1360782.

Naranjo Zolotov, M., Oliveira, T., Cruz-Jesus, F., and Martins, J., 2018a. Satisfaction with e-participation: A Model from the Citizen's Perspective, Expectations, and Affective Ties to the Place. In: Trends and Advances in Information Systems and Technologies. Cham: Springer International Publishing, pp. 1049-1059.

Naranjo Zolotov, M., Oliveira, T., and Casteleyn, S., 2018b. Continued intention to use online participatory budgeting: The effect of empowerment and habit. In: 11th International Conference on Theory and Practice of Electronic Governance.

Naranjo Zolotov, M., Oliveira, T., and Casteleyn, S., 2018c. Eparticipation adoption models research in the last 17 years: A weight and meta-analytical review. Computers in Human Behaviour, 81, 350-365. https://doi.org/10.1016/j.chb.2017.12.031

Pajarito, D., and Gould, M. (2017). Smart Mobility, the Role of Mobile Games. In: Serious Games (JCSG 2017). Springer, vol 10622, pp. 44-59.

Portela, M., and Granell-Canut, C. (2016). Methods to Observe and Evaluate Interactions with Everyday Context-Aware Objects. In: Ubiquitous Computing and Ambient Intelligence. Canary Islands, Spain, Nov 2016. Springer, vol. 10069, pp. 385-392.

Portela, M., and Granell-Canut, C. (2017a). A new friend in our Smartphone? Observing interactions with chatbots in the search of emotional engagement. In Proceedings of 18th International Conference Human Computer Interaction. Cancun, Mexico, Sep 2017. ACM, Article 48

Portela, M., and Granell-Canut, C. (2017b). A call to De-Familiarize with Everyday Objects: Understanding Modes of Ordering and Politics of Interaction. Presented at Digital Cities 10: Towards a Localised Socio-Technical Understanding of the 'Real' Smart City workshop, collocated with the Communities and Technologies conference 2017. Trolles, France, Jun 2017.

Portela, M., Acedo, A., and Granell-Canut, C. (2018). Looking for "in-between" places. Media Theory Journal, 2(1).

Roche, S., 2014. Geographic Information Science I: Why does a smart city need to be spatially enabled? Progress on Human Geography, 38, 703-711.

Smid, M., and Costa, A. C., 2017. Climate projections and downscaling techniques: a discussion for impact studies in urban systems. International Journal of Urban Sciences (in press), https://doi.org/10.1080/12265934.2017.1409132

Smid, M., Costa, A.C., Russo, S., Pebesma, E.J., and Granell C., 2017. Ranking of European Capitals according to the impact of Future Heat Waves. Presented at 2017 AGU Fall Meeting. New Orleans, Louisiana, Dec 2017
Van den Bergh, J.; Viaene, S., 2015. Key challenges for the smart city: Turning ambition into reality. In Proceedings of the 48th International Conference on System Sciences, Grand Hyatt, HI, USA, 5-8 January 2015.

World Economic Forum, 2017. Data Driven Cities: 20 Stories of Innovation. White paper 10 Nov 2017. https://www.weforum.org/whitepapers/data-driven-cities-20-stories-of-innovation (30 April 2018) 